\title{
Intensity of convective motions in marine atmospheric boundary layer retrieved from ocean surface radar imagery
}

\author{
M. I. Mityagina \\ Space Research Institute of Russian Academy of Sciences, Moscow, Russia \\ Received: 9 March 2006 - Revised: 30 June 2006 - Accepted: 1 July 2006 - Published: 24 July 2006
}

\begin{abstract}
The paper focuses on the occurrence and development of coherent structures observed in the atmosphere above ocean under natural conditions. Microwave imaging radars are suggested as data take instruments. The phenomena of marine atmospheric cells and rolls onset, horizontal planform, aspect ratio and scaling phenomena are examined. Convective patterns manifested in radar images and information derived on the intensity of atmospheric motion are discussed.
\end{abstract}

\section{Introduction}

Convective processes are literally the most frequently encountered types of motion of liquid and gas in the universe. This alone explains persistent and close interest of researchers to convection. Moreover, this attention has been recently encouraged due to another important fact: convection studies provide rich material for the exploration of new ideas concerning order and chaos equilibrium in hydrodynamics, simplicity and complexity of the structure and behavior of hydrodynamic objects. Convective flows can form more or less structured spatial patterns and their investigation helps to attain better understanding of general properties of pattern-forming systems. Convection properties are not only typical of various hydrodynamic instabilities, but also structure-forming processes of different nature.

Successful theoretical approaches must be firmly rooted in experiments. There are many examples in literature discussing results of laboratory studies of convective processes. However, there is much less information on observation of convective structures in natural conditions. Therefore, it is of interest to demonstrate the possibility to detect, observe

Correspondence to: M. I. Mityagina

(mityag@iki.rssi.ru) and explore convective structures in the atmosphere above ocean and indicate applicable methods and instruments.

The marine atmospheric boundary layer (MABL) is the part of the atmosphere that has direct contact and hence is directly influenced by the ocean. Thus, the MABL is where the ocean and atmosphere exchange large amounts of heat, moisture, and momentum, primarily via turbulent transport.

The ability to investigate convective motions in the atmosphere over the ocean using radar imagery of the ocean surface will be discussed in this paper.

From the standpoint of radar observation methods, the crucial peculiarities of the sea surface, as compared to the land, are its much lower scattering capability and physical homogeneity. Sea surface uniform dielectric properties greatly facilitate the analysis of radar images and allow direct association of radar backscattering signal with the geometric parameters of sea surface disturbances. Microwave radars sense the $\mathrm{cm}$-scale wave spectrum and, hence, $\mathrm{cm}$-scale sea surface roughness. Mesoscale atmospheric processes are known to affect sea surface roughness and hence to manifest themselves in radar images. Changes in wind speed and direction change the sea surface wave energy spectrum via the turbulent momentum flux between the atmosphere and ocean surface. Fine structures of the sea surface respond instantaneously to the wind. Because of various mechanisms of electromagnetic wave backscattering this is distinctly visible in radar signal modulations.

Samples of convective circulation and atmospheric eddies recorded by a radar with $3 \mathrm{~cm}$ wavelength are given in (Mitnik, 1992). A few less pronounced effects of such kind are seen in radar images of convective rolls and KelvinHelmholz waves recorded by a synthetic aperture radar with wavelength $5 \mathrm{~cm}$ mounted on European satellites ERS-1,2 (Alpers and Bruemmer, 1994).

A comparative analysis of radar images acquired in different bands revealed the Ku-band (wavelength $1.66-2.4 \mathrm{~cm}$ ) to be most sensitive to boundary layer conditions. The present

Published by Copernicus GmbH on behalf of the European Geosciences Union and the American Geophysical Union. 
Table 1. Main characteristics of the SARs mounted on the ERS and Envisat satellites.

\begin{tabular}{lll}
\hline Satellite & ERS-1/2 & Envisat \\
Sensor & SAR & ASAR \\
Frequency, GHz & 5.3 & 5.3 \\
Wavelength, cm & 5.6 & 5.6 \\
Polarization & VV & VV, HH \\
Incidence angle, deg & $20-26$ & $15-45$ (variable) \\
Swath width, km & 100 & $100-400$ \\
Ground resolution, m & $25 \times 25$ & $25 \times 25$ \\
& & $150 \times 150$ \\
\hline
\end{tabular}

work discusses the results of analysis and processing of a series of radar images of sea surface registered by a realaperture radar (RAR) with a wavelength of $2.25 \mathrm{~cm}$. Images were taken in conditions of warmer ocean surface compared to the near-surface layer of the atmosphere and manifested a variety of convection patterns.

\section{Instruments, methods and area of experiment - pre- liminary data analysis}

The data is obtained by airborne side-looking real aperture radar (RAR) mounted on TU-134 aircraft and by synthetic aperture radars (SARs) mounted on satellites ERS and Envisat (Bulatov et al., 2003).

The airborne radar is a Ku-band (wavelength $2.25 \mathrm{~cm}$ ) instrument operating at a peak power of $60 \mathrm{~kW}$, a $2 \mathrm{kHz}$ pulse repetition frequency and a $110 \mathrm{~ns}$ transmitted pulse width. Two cylindrical antennas, one on each side of the aircraft, transmit and receive alternate pulses of horizontal $(\mathrm{HH})$ and vertical (VV) polarization at large incidence angles of $72^{\circ}-$ $84^{\circ}$ to produce simultaneous $\mathrm{HH}$ and $\mathrm{VV}$ images. The radar illuminates a swath of $12.5 \mathrm{~km}$ on each side of the ground track with a spatial resolution of about $25 \times 25 \mathrm{~m}$.

Main characteristics of the SARs mounted on the ERS and Envisat satellites are summarized in Table 1.

Results are based on the analysis of a series of field experiments conducted in North Pacific in 1981, 1985 and 1987 (August-September) as well as in Northwest Atlantic during the Joint US/Russia Experiment in 1992 (July) when radar measurements of convection processes were made. Radar images obtained over the Black Sea and Baltic Sea in 19992005 are also considered. The analysis involved meteorological parameters measured in situ over the corresponding periods.

Extensive analysis of numerous images recorded under stable, unstable and neutral MABL conditions shows that cell-shaped or roll-shaped signatures are distinctly visible in VV radar images every time when the sea surface is warmer than the near-surface air. These patterns are related to the

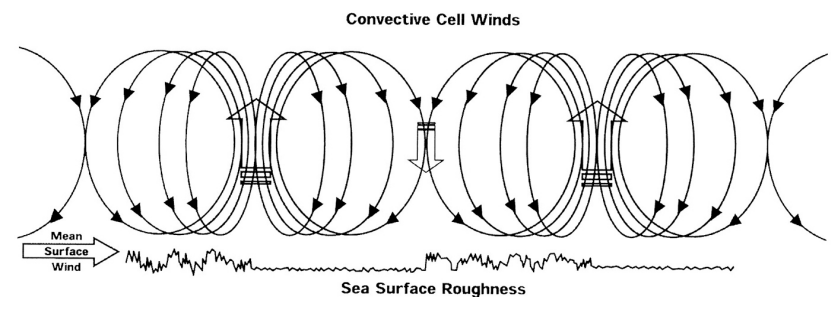

Fig. 1. Vertical cross section through an atmospheric convective cell above ocean.

origin and development of atmospheric convection resulted from unstable stratification in the ocean-atmosphere boundary layer. Thus, cells and rolls over the ocean are coherent structures of enhanced air-sea interaction. Each of them has distinctive signatures on the ocean surface roughness. These signatures are the result of the alteration of the ocean surface on the cm-scale that results from gusts of wind emanating from the ocean downdrafts of the coherent structures. These gusts result from the mixing of high momentum air from aloft downwards toward the sea surface. At the same time the updrafts tend to transport low-momentum air upwards. Thus, both of these processes cause negative turbulent momentum flux. Figure 1 shows a cell-induced (perturbation) flow pattern and the resulting sea surface roughness after interaction with the mean surface wind. Sea surface roughness (and resulting radar backscatter) increases when the cell winds and mean surface wind are in the same direction and diminishes when they are opposed.

Typical dimensions of convective structures in the plane of observation are 800-1500 $\mathrm{m}$ for the Pacific test region and 1000-2000 m for the Northwest Atlantic. Approximately $70 \%$ of the images bearing surface imprints of MABL cell convection were obtained in the absence of clouds. The regular spatial structures seen in the images effectively preceded the formation of convective clouds.

\section{Effects of atmospheric stability on radar image pat- terns}

\subsection{Qualitative analysis}

Perhaps the most striking aspect of radar imagery under high viewing angles is the extreme sensitivity of the verticalpolarization clutter characteristics to atmospheric stability (Mityagina et al., 1998; Ufermann and Romeiser, 1999).

Under stable atmospheric conditions, when the air temperature is greater than the surface one, the vertical polarization images are qualitatively similar to those recorded at horizontal polarization. Radar images in Fig. 2a obtained on 19 July 1992 illustrate this fact. Surface manifestations of internal waves are distinctly seen both at $\mathrm{HH}$ and VV polarizations, although VV contrasts are less pronounced. 


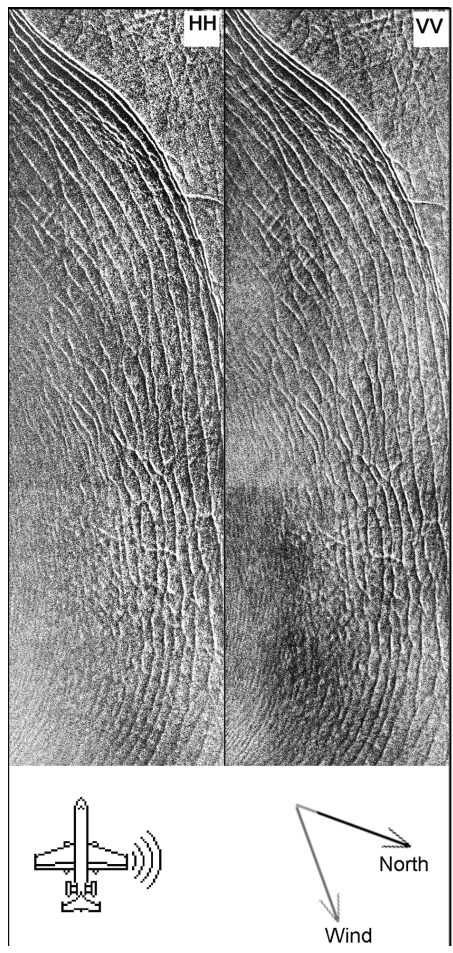

(a)

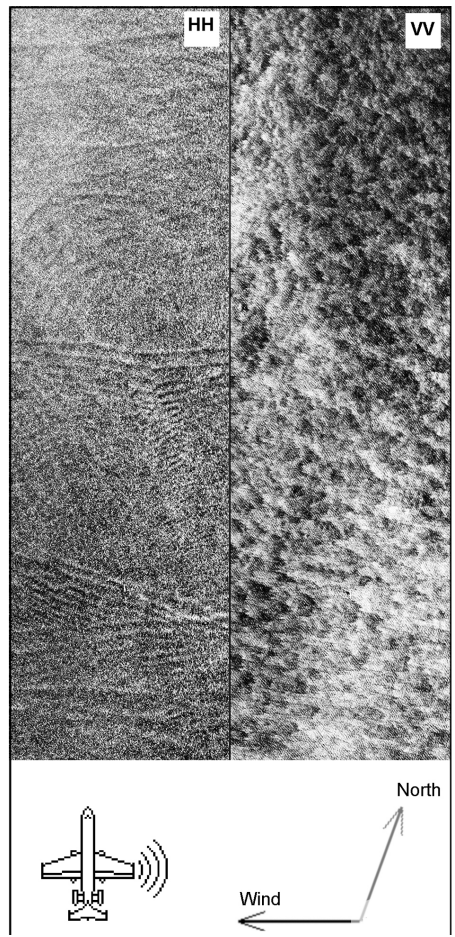

(b)

Fig. 2. Dual polarization (VV and $\mathrm{HH}$ ) radar images of sea surface obtained under (a) stable atmosphere boundary layer conditions on 19 July 1992; (b) unstable atmosphere boundary layer conditions on 16 July 1992.

However, under unstable atmospheres the situation is quite opposite. Near-surface wind variations induced by intensive
Table 2. Min and Max values of 2D-correlation function of VV and $\mathrm{HH}$ polarized images of sea surface under stable and unstable atmospheres.

\begin{tabular}{cccccccc}
\hline \multicolumn{3}{c}{ Stable conditions } & \multicolumn{5}{c}{ Unstable conditions } \\
$\begin{array}{c}\text { Wind } \\
\text { Speed } \\
(\mathrm{m} / \mathrm{s})\end{array}$ & $\begin{array}{c}\mathrm{T}_{\text {air }}-\mathrm{T}_{\text {water }} \\
{ }^{\circ} \mathrm{C}\end{array}$ & Min & Max & $\begin{array}{c}\text { Wind } \\
\text { Speed }\end{array}$ & $\begin{array}{c}\mathrm{T}_{\text {air }}-\mathrm{T}_{\text {water }} \\
{ }^{\circ} \mathrm{C}\end{array}$ & Min & Max \\
\hline $3-5$ & 6 & -0.16 & 0.63 & $3-5$ & -2 & -0.123 & 0.1 \\
$6-7$ & 5 & -0.24 & 0.41 & $5-6$ & -2.5 & -0.04 & 0.2 \\
$5-6$ & 6 & -0.06 & 0.43 & $6-8$ & -1.5 & -0.04 & 0.17 \\
$3-5$ & 4 & -0.16 & 0.65 & $4-6$ & -2 & -0.1 & 0.1 \\
$5-6$ & 5 & -0.07 & 0.49 & $2-4$ & -4 & -0.02 & 0.08 \\
$6-7$ & 5 & -0.27 & 0.59 & $2-4$ & -3.5 & -0.05 & 0.09 \\
8 & 6 & -0.13 & 0.60 & $5-7$ & -2 & -0.02 & 0.18 \\
\hline
\end{tabular}

convection in the boundary layer produce high contrast cellular pattern in the VV images, while HH-polarized ones remain undisturbed. Sometimes the images at $\mathrm{HH}$ and VV polarization look so different that it is hard to believe they come from the same scene. Under conditions of unstable stratification of the marine atmospheric boundary layer, some images exhibit clear signatures of oceanic internal waves at $\mathrm{HH}$ polarization, while the corresponding VV images are dominated by signatures of wind variations associated with atmospheric convective cells. HH and VV sea surface radar images obtained on 16 July 1992 under unstable stratification of the MABL are presented in Fig. 2b. Near-surface wind variations induced by intensive convection in the boundary layer produced high contrast cellular pattern in the VV image. The typical size of these cells is about $1.5-2 \mathrm{~km}$.

These phenomena are observed in radar images recorded over a number of years of experiments in the Northwest Pacific, near the Kamchatka peninsula. Numerous images recorded under stable, unstable and neutral atmospheric boundary layer conditions show that cellular-type mottled signatures in VV radar images are distinctly visible every time when the sea surface is warmer than the near surface air and are never detected under other conditions. This means that the analysis of radar images at vertical polarization makes it possible to determine the type of the boundary layer stratification and radar images clutter patterns at vertical polarization can be regarded as an indicator of boundary layer conditions.

As shown by (Churyumov et al., 2002), the two polarizations sense different scatterers at unstable stratification: horizontally polarized radiation scatters predominantly from steep waves, while vertical polarization scatters primarily from free Bragg waves. The fact that steep surface waves are produced mostly by oceanic gravity waves, while free Bragg waves are generated by atmospheric motion enables us to separate surface manifestations of oceanic and atmospheric processes in radar images. 


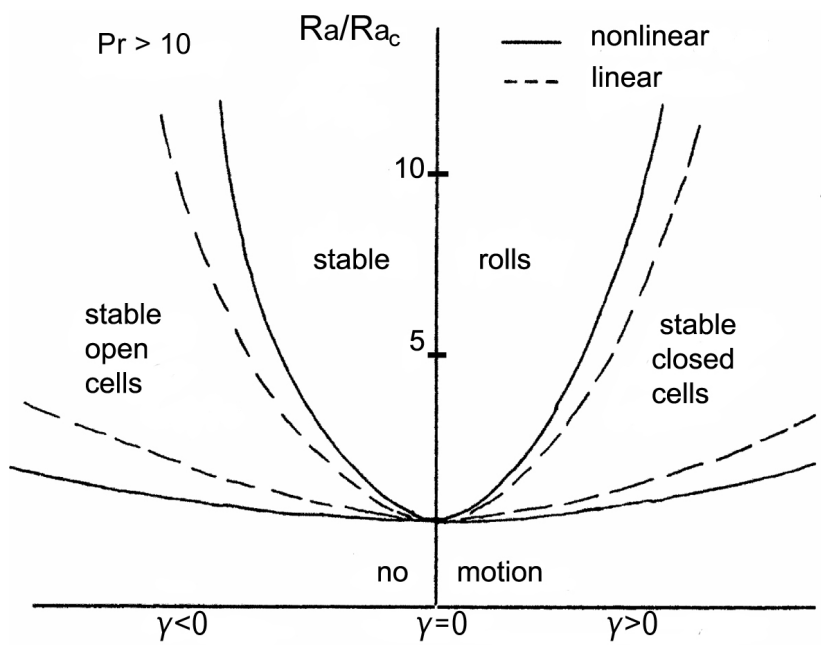

Fig. 3. Schematic of regime stability diagram for the onset of thermal convection (after Krishnamurti, 1975).

\subsection{Quantitative estimates}

Thus, VV and HH polarization images are associated with different scattering mechanisms and radar images at two polarizations yield significantly different backscatter information. VV polarization often senses effects in the atmosphere over the ocean, while HH polarization is sensitive to processes in the sea itself. Hence, the detection of the type of the ocean-atmosphere boundary layer may be based on the polarization differences of the radar cross section.

The 2-D-correlation functions of $\mathrm{VV}$ and $\mathrm{HH}$-polarized radar images recorded by the above mentioned airborne dual-polarizations radar were considered with the aim to state a quantitative criterion for the identification and discrimination between stable, unstable and neutral oceanatmosphere stratifications. 2-D-correlation functions for fragments $(10 \times 10 \mathrm{~km})$ of simultaneous VV and HH polarized images of sea surface were calculated. Table 2 gives the limits of variations of 2-D correlation functions for HH-VV pairs of images taken under stable and unstable conditions. Maximums of absolute values do not exceed 0.2 in unstable conditions and are greater then 0.4 in stable conditions. We believe the 2-D-correlation criterion to be rather simple and reliable.

\section{Detection of convection regime in the atmosphere over the ocean}

For the interpretation of experimental data a model presented in (Krishnamurti, 1975) was used. This model is based on hydrodynamical equations in Boussinesq approximation. The model introduces an atmospheric Rayleigh number $R a$ for dry convection, where the thermal conductivity and the molecular viscosity are replaced by their eddy counterparts.
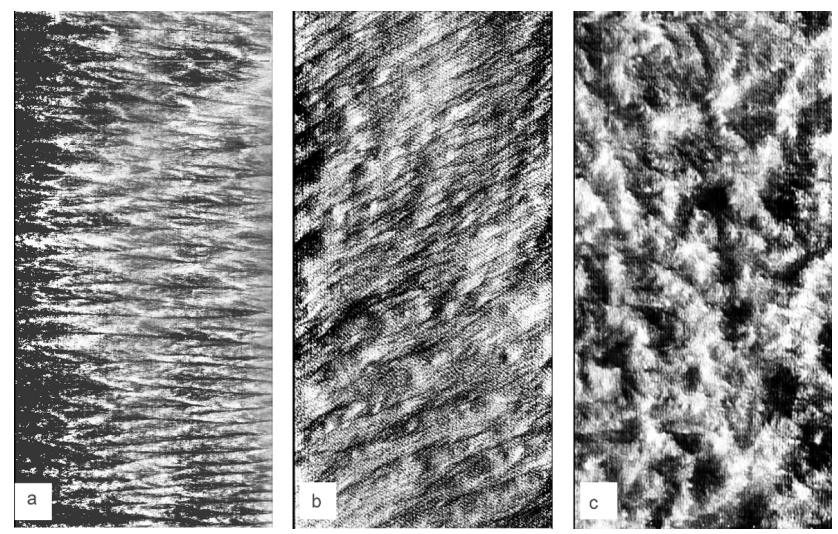

Fig. 4. Ku-band RAR images featuring different regimes of atmospheric convection. Images are registered in North-Western Pacific near Kamchatka peninsula. (a) convective rolls, $\Delta \mathrm{T}=-0.4^{\circ}$, wind speed $=6 \mathrm{~m} / \mathrm{s}$; (b) convective rolls and cells, $\Delta \mathrm{T}=-1.5^{\circ}$, wind speed $=7.5 \mathrm{~m} / \mathrm{s} ;$ (c) convective cells, $\Delta \mathrm{T}=-6.5^{\circ}$, wind speed $=6 \mathrm{~m} / \mathrm{s}$.

Krishnamurti extended this type of formulation for moist convection, and determined the regime-stability diagram for both linear and non-linear model equations under conditions of weak supercritical $R a$. Her findings are shown schematically in Fig. 3, where the anisotropy parameter $\gamma$ represents vertical motion. As seen in Fig. 3, if the fluid is initially at rest $(\gamma=0)$ or moving with constant horizontal velocity, the convective shape at the onset is an aggregation of two-dimensional stable rolls. These patterns in convective marine boundary layers have at the onset a typical aspect ratio (wavelength-to-depth) of about 3 to 1 . If there is large-scale vertical motion the onset shape becomes threedimentional and occurs as stable array of hexagonal cellular convection. Krishnamurti also showed that the sign of the large-scale vertical velocity controlled the circulation direction, with up motion $(\gamma>0)$ producing closed cells and down motion $(\gamma<0)$ producing open cells.

The results of our field experiments were considered and compared with the regime stability diagram shown in Fig. 3. Figure 4 features a set of vertical polarization radar images of sea surface obtained over the North Pacific near Kamchatka peninsula. The time and coordinates of observations are given for each image. Wind speed magnitudes and directions as well as temperature differences of atmosphere and ocean are also indicated. The images contain imprints of various convective patterns.

Convection processes caused by weak thermodynamical instability of atmosphere (small dT/dz) and by slow cooling rate of sea surface occur as roll-formed flow. Hence, radar signatures of convective flows under linear temperature profile and weak instability should cause the appearance of alternating bands of weak and strong backscattering. Figure $4 \mathrm{a}$ illustrates this situation. 
When the atmospheric instability grows stronger, the cylinder structure of flows changes into a cell structure. This situation is shown in Fig. 4b. The precise threshold conditions for this translation are still the topic for theoretical discussions. However, radar imagery may be employed to study this transition in unprecedented spatial detail and without the need for the existence of clouds that many previous studies have relied on.

A system of well-developed convective cells exists under conditions of strong atmospheric instability. These cells can be seen in Fig. 4c. It is worth to mention that surface imprints of atmospheric convective cells are often visible in radar images of sea surface taken under unstable atmospheres, while observations of roll structures are less frequent.

We conclude that radar backscattering from ocean surface can supply information about vertical motion and energy exchange in the MABL. While cylinder-shaped structures are quite visible in radar images, vertical motions and energy exchange in the MABL are expressed rather weakly. On the other hand, cellular clutter patterns can be regarded as an indicator of prominent vertical motions in the boundary layer, with their horizontal sizes growing with the increase of the temperature difference $T_{\text {water }}-T_{\text {atm }}$.

\section{Regular deformations of convective structures}

As a rule, convective patterns produced in radar images differ from regular forms predicted by theory.

Figure 5a presents an ERS-2 SAR image recorded on 15 September 1999 over the east-northern part of the Black Sea. The mean surface wind speed was $11 \mathrm{~m} / \mathrm{s}$, wind direction $10^{\circ}$. The temperature difference $\Delta \mathrm{T}=\mathrm{T}_{\mathrm{atm}}-\mathrm{T}_{\text {water }}$ was $-6.5^{\circ} \mathrm{C}$. Cellular patterns distinctly seen in this image look like deformed polygons. The asymmetry of observed cells is explained by the presence of a mean flow in the horizontal plan view resulting in the asymmetry of weak and strong backscatter zones.

Figure $5 \mathrm{~b}$ shows the result of a numerical simulation. A hexagonal cell on a horizontal plane is schematically presented. The density of the dot shading is the indicator of the downward velocity component increase. The denser the dots the larger is the downward vertical velocity. Within the central circle, velocity is upward.

Two-dimensional spatial Fourier spectrum of a fragment of the SAR image is depicted in Fig. 5c. It proves the existence of the hexagonal asymmetry of convective cells seen in radar images of the sea surface. In natural conditions, air circulation in cylinder- or cell-shaped structures differs from the model. In particular, the cell form is distorted due to general transference, interactions between circular flows in neighboring cells, combinations of cells and rolls, etc. This implies a variety of radar patterns of convective processes occurring in the MABL, which has to be considered when interpreting the images. Near-surface wind variations due to convection

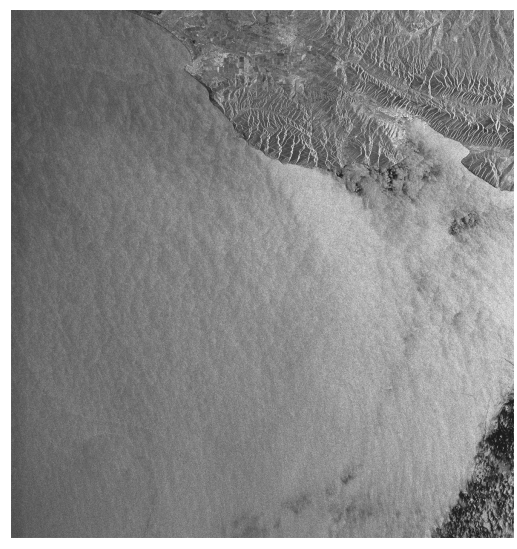

(a)

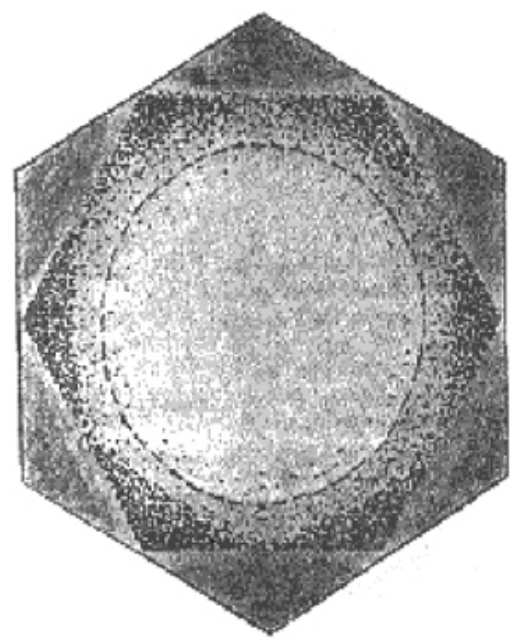

(b)

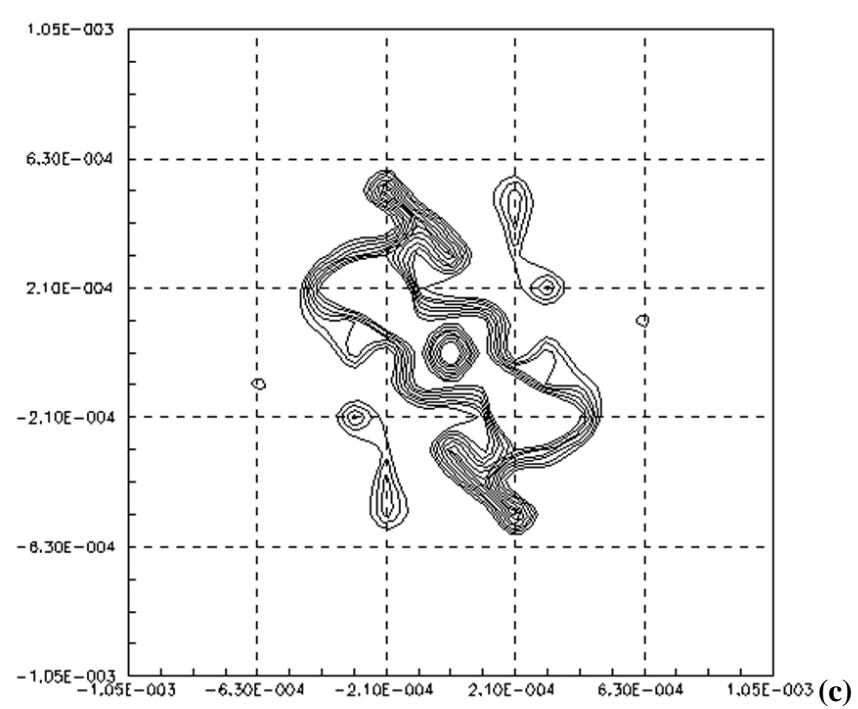

Fig. 5. (a) ERS-2 SAR image recorded on 15 September 1999 over the Black Sea; (b) Hexagonal convective cell on a horizontal plane (numerical modeling); (c) Two-dimensional spatial Fourier spectrum $\left(\mathrm{m}^{-1}\right)$ of the fragment of the SAR image bearing imprints of convective cells. 
leave a clear imprint in radar images, in the shape of bands of alternating intensity in the case of roll convection, and in the shape of round or sickle-shaped structures coinciding with some cells in the case of cell convection.

\section{Conclusions}

The possibility to distinguish and investigate large-scale vertical motions in the near-surface atmospheric layer by means of radar imagery is substantiated.

Knowledge of the spatial and temporal distribution as well as intensity of cells and rolls is particularly important. The existence and the type of coherent structures in the atmosphere over the ocean tell the analyst much about the larger scale atmospheric motions. In principal, average convection properties that are often necessary to know (first of all, heat flow transported by convective liquid) can be derived from the form and scale of the current. Airborne and space borne microwave imaging radars are effective instruments for the study of physical processes occurring in the MABL, including convection. The extensive use of these radars will increase the amount of information registered by remote sensing systems.

Acknowledgements. The author expresses deep gratitude to S. S. Moiseev and V. G. Pungin for inspiring the conducted research and useful discussions.

Edited by: N. S. Erokhin

Reviewed by: four referees

\section{References}

Alpers, W. and Bruemmer, B.: Atmospheric boundary layer rolls observed by the synthetic aperture radar aboard the ERS-1 satellite, J. Geophys. Res., 99, 12 613-12 621, 1994.

Bulatov, M. G., Kravtsov, Yu. A., Lavrova, O. Yu., Litovchenko, K. Ts., Mityagina, M. I., Raev, M. D., Sabinin, K. D., Trokhimovskii, Yu. G., Churyumov, A. N., and Shugan, I. V.: Physical mechanisms of aerospace radar imaging of the ocean, PhysicsUspekhi, 46(1), 63-79, 2003.

Churyumov, A. N., Kravtsov, Yu. A., Lavrova, O. Yu., Litovchenko, K. Ts., Mityagina, M. I., and Sabinin, K. D.: Signatures of resonant and non-resonant scattering mechanisms on radar images of internal waves, Int. J. Remote Sens., 23(20), 4341-4355, 2002.

Krishnamurti, R.: On cellular cloud patterns, J. Atmos. Sci., 33, 1353-1383, 1975.

Mitnik, L. M.: Mesoscale coherent structures in the surface wind field during cold air outbreaks over the Far Eastern seas from the satellite side looking radar, La Mer, 30, 287-296, 1992.

Mityagina, M. I., Pungin, V. G., and Yakovlev, V. V.: Twopolarization $\mathrm{K}_{u}$-band radar imagery of sea surface in presence of atmospheric boundary layer motions, Waves in Random Media, 8, 111-118, 1998.

Ufermann, S. and Romeiser, R.: Numerical study on signatures of atmospheric convective cells in radar images of the ocean, J. Geophys. Res., 104, 25 707-25 720, 1999. 